\title{
Calculation of critical values of several probability distributions using standard numerical methods
}

\author{
Dušan Knežo ${ }^{1}$, Jozef Zajac ${ }^{2}$, Peter Michalik ${ }^{3}$
}

\begin{abstract}
Very common statistical methods used in practice are interval estimations, tests of statistical hypotheses and other tests. Using these methods usually requires knowledge of critical values of some distributions. In the case of mentioned application methods are used computer programs, but in other cases it is desirable to have a method of calculating the distribution of the critical values. Article presents methods for calculating the critical values for a standard normal distribution, Student's t-distribution, Fisher-Snedecor F-distribution and $\chi^{\wedge} 2$ distribution using standard numerical methods, specifically an approximate calculation of definite integrals and approximate calculation of nonlinear equations.
\end{abstract}

Keywords: probability distributions, critical values, approximate calculation of definite integrals, approximate calculation of nonlinear equations.

\section{Introduction}

Application of some statistical methods for example interval estimations or statistical hypothesis tests, which requires some critical values probability distribution, particularly a standard normal distribution, Student's $t$-distribution, FisherSnedecor $F$-distribution and $\chi^{2}$-distribution.

If the function $F(x)$ is distribution function of the probability distribution of the random variable $X$, thus the critical value of this distribution to the significance level $\alpha$ is the value $x_{\alpha}$, to which is valid

$$
P\left(X>x_{\alpha}\right)=1-F\left(x_{\alpha}\right)=\alpha .
$$

Determining critical values is usually made by using tables or specialized statistical software. This approach also has its disadvantages, but in some cases is advantageous to have a method by which is possible to calculate evaluated critical values. Calculation of critical values is also possible using standard numerical methods, specifically method of approximate calculation of definite integrals and methods of approximate calculation of nonlinear equations. Analogical uses of methods are presented in article according [1-3].

1 Technical University of Košice, Faculty of manufacturing technologies with a seat in Prešov, Department of mathematics, informatics and cybernetics, Bayerova 1, 08001 Prešov, Slovak Republic, email: dusan.knezo@tuke.sk

2 Technical University of Košice, Faculty of manufacturing technologies with a seat in Prešov, Department of computer aided manufacturing technologies, Štúrova 31, 08001 Prešov, Slovak Republic, email: jozef.zajac@tuke.sk

3 Technical University of Košice, Faculty of manufacturing technologies with a seat in Prešov, Department of manufacturing technologies, Štúrova 31, 08001 Prešov, Slovak Republic, email: peter.michalik@tuke.sk 
Study [2] prescribes methods for determination critical values of standard normal and Student's t-distribution.

Equation (1) is used to determinate critical values $x_{\alpha}$ of standard normal distribution $\mathrm{N}(0,1)$ in the level of significant $\alpha$ and implies (Fig. 1)

$$
1-\phi\left(x_{\alpha}\right)=\alpha,
$$

where $\phi(x)$ is distribution function of standard normal distribution $\mathrm{N}(0,1)$ and is define by equation

$$
\phi(x)=\int_{-\infty}^{x} \varphi(t) d t=\frac{1}{\sqrt{2 \pi}} \int_{-\infty}^{x} e^{-\frac{t^{2}}{2}} d t
$$

From the properties of the distribution function $\varphi(x)$ implies that

$$
\phi(x)=\left\{\begin{array}{cl}
0.5-\frac{1}{\sqrt{2 \pi}} \int_{0}^{|x|} e^{-\frac{t^{2}}{2}} d t, & x<0, \\
0.5, & x=0, \\
0.5+\frac{1}{\sqrt{2 \pi}} \int_{0}^{x} e^{-\frac{t^{2}}{2}} d t, & x>0 .
\end{array}\right.
$$

From equation (4) can be state, that is je is sufficient to limit consideration to the integral

$$
\int_{0}^{x} \varphi(t) d t=\frac{1}{\sqrt{2 \pi}} \int_{0}^{x} e^{-\frac{t^{2}}{2}} d t
$$

valid for $x>0$. Calculating integral (5) can be used Simpson's rule and can be deduces

$$
\left|\varphi^{(4)}(t)\right| \leq \varphi^{(4)}(0)=\frac{3}{\sqrt{2 \pi}}
$$

For integration step $h$ of approximate calculation of integral (5) using Simpson's rule with accuracy $\varepsilon$ implies

$$
h \leq \sqrt[4]{\frac{60 \cdot \varepsilon \cdot \sqrt{2 \pi}}{x}} .
$$

Based on previous equations is possible with accuracy $\varepsilon$ to calculate values of the distribution function, thus is possible to set critical value $x_{\alpha}$ with required accuracy to evaluate equation

$$
\phi\left(x_{\alpha}\right)+\alpha-1=0
$$

Because function 


$$
G(x)=\phi(x)+\alpha-1
$$

is increasing continuous function,

$$
\lim _{x \rightarrow-\infty} G(x)=\alpha-1<0
$$

and

- $\lim _{x \rightarrow \infty} G(x)=\alpha>0$,

then equation (8) has exactly one solution. Approximate solution of equation (8) can be done using bisection method.

Table 1 shows some critical values of standard normal distributions calculated by described method.

Probability density according to Students $t$-distribution with $n$ degrees of freedom is evident, that distribution function can be define as follows

- $F_{n}(x)= \begin{cases}0.5-c_{n} \cdot \int_{0}^{|x|}\left(1+\frac{t^{2}}{n}\right)^{-\frac{n+1}{2}} d t, & x<0 \\ 0.5, & x=0 \\ 0.5+c_{n} \cdot \int_{0}^{x}\left(1+\frac{t^{2}}{n}\right)^{-\frac{n+1}{2}} d t, & x>0\end{cases}$

where

$$
c_{n}=\frac{\Gamma\left(\frac{n+1}{2}\right)}{\sqrt{n \pi} \cdot \Gamma\left(\frac{n}{2}\right)} .
$$

For approximate integral calculation

$$
\int_{0}^{x} g_{n}(t) d t=\int_{0}^{x}\left(1+\frac{t^{2}}{n}\right)^{-\frac{n+1}{2}} d t
$$

for $x>0$ can be used trapezoidal rule. Can be demonstrated

$$
\left|g_{n}^{\prime \prime}(t)\right| \leq g_{n}^{\prime \prime}(0)=\frac{n+1}{n} .
$$

For step of approximate integral calculation (14) using trapezoidal rule with accuracy $\varepsilon$ is valid

$$
h \leq \sqrt{\frac{12 n \varepsilon}{(n+1) \cdot x}} .
$$


Determination of critical value $x_{\alpha}$ by Students $t$-distribution (Fig. 2) is to find the equation root

$$
1-F_{n}(x)=\alpha
$$

Approximate solution of equation (17) can be realized using bisection method. Table 2 shows some critical values of Student $\mathrm{t}$-distribution with $\mathrm{n}$ degrees of freedom calculated by described method.

\section{Critical values of $\chi^{2}$-distribution}

Probability density $\chi^{2}$-distribution with $n$ degrees of freedom is define by equation

$$
f_{n}(x)= \begin{cases}\frac{1}{2^{\frac{n}{2}} \cdot \Gamma\left(\frac{n}{2}\right)} \cdot x^{\frac{n}{2}-1} \cdot e^{-\frac{x}{2}}, & x>0 \\ 0, & x \leq 0\end{cases}
$$

Because for $n \in N$ and $n \geq 2$ for calculation values of function $\Gamma$ can be used equation

$$
\Gamma(k)=(k-1) !
$$

or

$$
\Gamma\left(k+\frac{1}{2}\right)=\frac{1 \cdot 3 \cdot 5 \cdots(2 k-1)}{2^{k}} \cdot \sqrt{\pi}
$$

where $k \in N$.

Equation (1) for determinate critical value $x_{\alpha} \chi^{2}$-distribution (Fig. 3) with $n$ degrees of freedom on confidence level $\alpha$ is apparent

$$
1-F_{n}(x)=\alpha,
$$

where $F_{n}(x)$ is distribution function define by equation

$$
F_{n}(x)=\int_{0}^{x} f_{n}(t) d t
$$

Derivation properties of function $f_{n}(x)$ shows, that in general is not possible to define step for approximate integral calculation (22) to obtain required accuracy. In that case is approximate integral calculation (22) would be appropriate to use 
Richardson extrapolation and Simpson's method. Calculation uncertainty/error $E$ is estimate by equation

$$
|E| \cong \frac{\left|I_{h}-I_{h / 2}\right|}{15},
$$

where $I_{s}$ is approximate value of the integral calculated by the Simson's method with using step $s$.

Equation (21) has exactly one solution and its approximate values can be found using bisection method. Table 3 shows some critical values for $\chi^{2}$-distribution with $n$ degrees of freedom calculated using described evaluation method.

\section{Critical values of $\boldsymbol{F}$-distribution}

The probability density of $F$-distribution with $m, n$ degrees of freedom is in interval $[0, \infty)$ prescribed by equation

$$
f_{m, n}(x)=c_{m, n} \cdot \frac{x^{\frac{m}{2}-1}}{(m x+n)^{\frac{m+n}{2}}},
$$

where

$$
c_{m, n}=\frac{\Gamma\left(\frac{m+n}{2}\right) \cdot m^{\frac{m}{2}} \cdot n^{\frac{n}{2}}}{\Gamma\left(\frac{m}{2}\right) \cdot \Gamma\left(\frac{n}{2}\right)} .
$$

Because $m, n \in N$ values of function $\Gamma$ can be calculated using equations (19) and (20).

Equation (1) for determination of critical values $x_{\alpha}$, subsequently $F$ distribution with $m, n$ degrees of freedom on significant level $\alpha$ is apparent (Fig. 4).

$$
1-F_{m, n}\left(x_{\alpha}\right)=\alpha,
$$

where $F_{m, n}(x)$ is distribution function and is define by equation

$$
F_{m, n}(x)=\int_{0}^{x} f_{m, n}(t) d t .
$$

Analogy with the previous section, in general is not possible to determined step of approximate integral calculation (27) to obtain a specified accuracy. In this case it is also appropriate to use Richardson extrapolation method and Simpson's method. 
Equation (26) has exactly one solution and approximate value can be possible find using bisection method. Table 4-7 list some of critical values of $F$-distribution with $m, n$ degrees of freedom calculated using prescribed method.

\section{Conclusions}

Described methods present possibilities of calculation critical values for the most common probability distributions used in practice. Advantage of these methods is that they can be used as part of automatic data processing, e.g. at interval estimates or statistical testing. Advantageous is that they allows the calculation of the critical values for any significance level. Methods can also be used to create tables included in books and textbooks in the field of statistics. Program for each of described methods was designed in a language Free Pascal - Lazarus IDE. Algorithms of these programs are of course usable in other programming languages. Application of described methods is limited with technical possibilities of computer, because at some values computer works at machine's boundary limits. Described methods are based on standard numerical methods of calculation definite integrals. Other methods such as Monte Carlo and others can be used as well.

\section{Acknowledgements}

This work is a part of research project VEGA 1/0619/15. The article was prepared within the invitation OPVaV-2012/2.2/08-RO named "University Science Park TECHNICOM for Innovation Applications with the Support of KnowledgeBased Technologies" code ITMS 26220220182 and of the project VEGA 1/0594/12.

\section{References}

[1] KNEŽO, D. (2014) Inverse transformation method for normal distribution and the standard numerical method. International Journal of Interdisciplinarity in Theory and Practice 5: 6-10.

[2] KNEŽO, D. (2014) Application of the numerical methods for the teaching of the statistics. International Journal of Interdisciplinarity in Theory and Practice 5: 58-61.

[3] KNEŽO, D. (2012) O metóde Monte Carlo a možnostiach jej aplikácií. Transfer inovácií 24: 178-181.

[4] BONNAR J. (2014) J. Bonnar, The Gamma Function (CreateSpace).

[5] WALCK CH. (2007) Hand-book on statistical distributions for experimentalist (Universitet Stockholms). 
[6] KOKOSKA S., NEVISON CH. (1992) Statistical Tables and Formulae (New York: Springer-Verlag).

[7] NEAVE H. R. (1981) Elementary Statistics Tables (London: George Allen \& Unwin).

[8] NEAVE H. R. (2011) Elementary Statistics Table (London: Routledge).

[9] NEAVE H. R. (2011) Statistics Tables: For Mathematicians, Engineers, Economists and the Behavioural and Management Sciences (London: Routledge) 
Figure 1. Critical values of standard normal distribution [6]

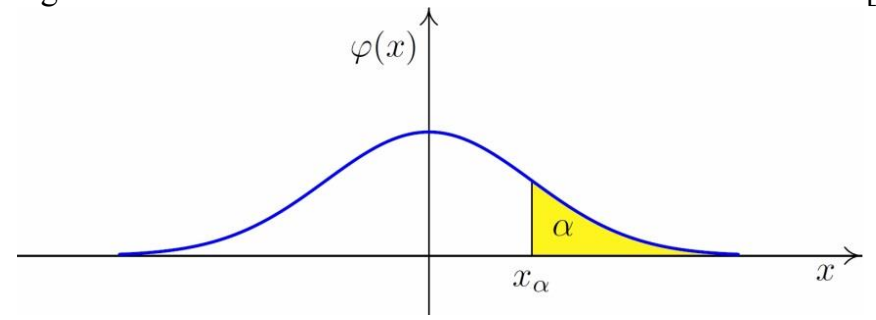

Figure 2. Critical values of Students $t$-distribution [7]

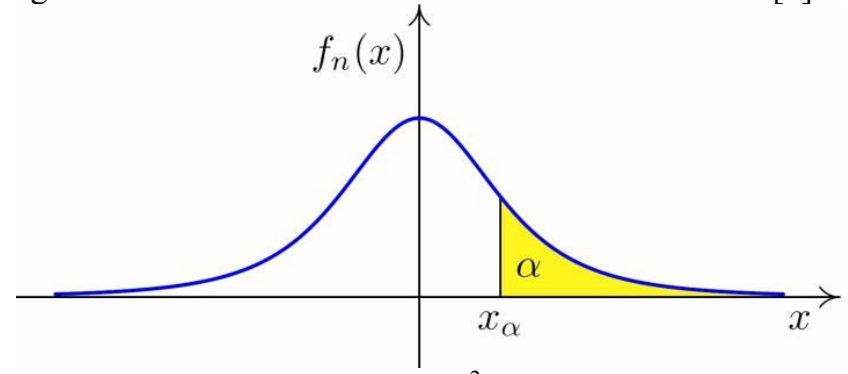

Figure 3. Critical values of $\chi^{2}$-distribution

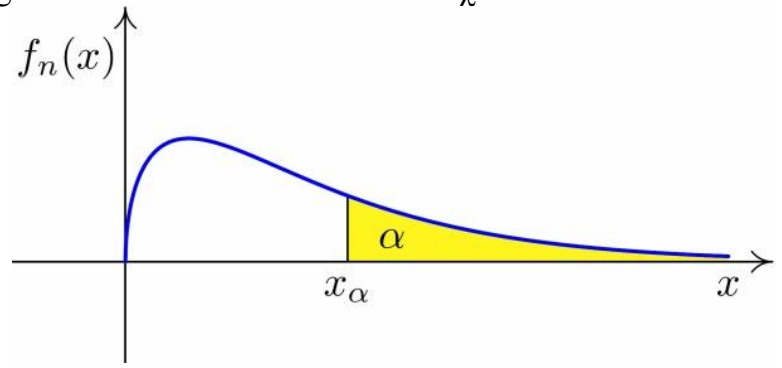

Figure 4. Critical values of F-distribution

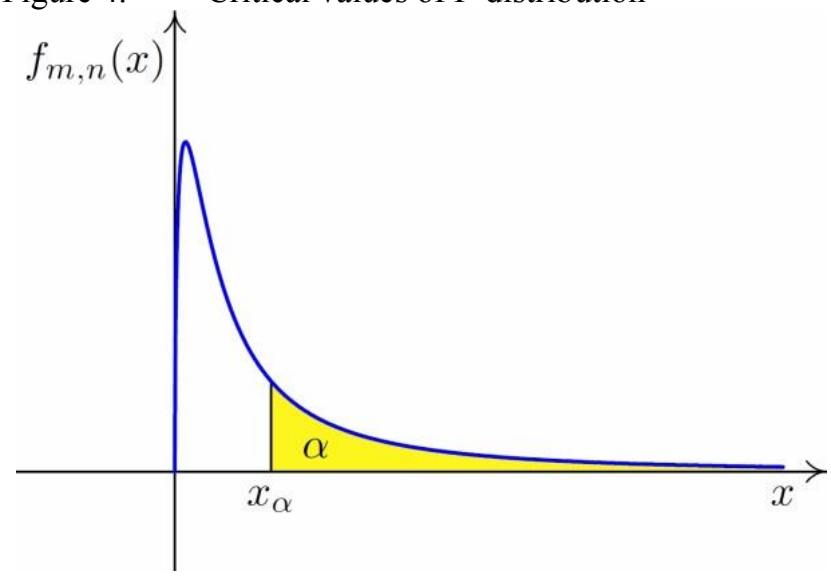


Table 1. Selected critical values of standard normal distribution

\begin{tabular}{lllllll}
\hline$\alpha$ & 0.10 & 0.05 & 0.025 & 0.0125 & 0.01 & 0.005 \\
$x_{\alpha}$ & 1.281551 & 1.644854 & 1.959965 & 2.241401 & 2.326350 & 2.575833 \\
\hline
\end{tabular}

Table 2. Selected critical values of Students $t$-distribution

\begin{tabular}{lrrrrr}
\hline$n$ & $\alpha=0.1$ & $\alpha=0.05$ & $\alpha=0.025$ & $\alpha=0.01$ & $\alpha=0.001$ \\
\hline 3 & 1.638 & 2.353 & 3.182 & 4.541 & 10.215 \\
4 & 1.533 & 2.132 & 2.776 & 3.747 & 7.173 \\
5 & 1.476 & 2.015 & 2.571 & 3.365 & 5.893 \\
6 & 1.440 & 1.943 & 2.447 & 3.143 & 5.208 \\
7 & 1.415 & 1.895 & 2.365 & 2.998 & 4.785 \\
8 & 1.397 & 1.860 & 2.306 & 2.896 & 4.501 \\
9 & 1.383 & 1.833 & 2.262 & 2.821 & 4.297 \\
10 & 1.372 & 1.812 & 2.228 & 2.764 & 4.144 \\
11 & 1.363 & 1.796 & 2.201 & 2.718 & 4.025 \\
12 & 1.356 & 1.782 & 2.179 & 2.681 & 3.930 \\
13 & 1.350 & 1.771 & 2.160 & 2.650 & 3.852 \\
14 & 1.345 & 1.761 & 2.145 & 2.624 & 3.787 \\
15 & 1.341 & 1.753 & 2.131 & 2.602 & 3.733 \\
16 & 1.337 & 1.746 & 2.120 & 2.583 & 3.686 \\
17 & 1.333 & 1.740 & 2.110 & 2.567 & 3.646 \\
18 & 1.330 & 1.734 & 2.101 & 2.552 & 3.611 \\
19 & 1.328 & 1.729 & 2.093 & 2.539 & 3.579 \\
20 & 1.325 & 1.725 & 2.086 & 2.528 & 3.552 \\
21 & 1.323 & 1.721 & 2.080 & 2.518 & 3.527 \\
22 & 1.321 & 1.717 & 2.074 & 2.508 & 3.505 \\
23 & 1.319 & 1.714 & 2.069 & 2.500 & 3.485 \\
\hline & & & & &
\end{tabular}

Table 3. Selected critical values of $\chi^{2}$-distribution

\begin{tabular}{cccccc}
\hline$n$ & $\alpha=0.1$ & $\alpha=0.05$ & $\alpha=0.025$ & $\alpha=0.01$ & $\alpha=0.001$ \\
\hline 3 & 6.251 & 7.815 & 9.348 & 11.345 & 16.267 \\
4 & 7.779 & 9.488 & 11.143 & 13.277 & 18.467 \\
5 & 9.236 & 11.070 & 12.832 & 15.086 & 20.515 \\
6 & 10.645 & 12.592 & 14.449 & 16.812 & 22.458 \\
7 & 12.017 & 14.067 & 16.013 & 18.475 & 24.322 \\
\hline
\end{tabular}


10

\begin{tabular}{llllll}
8 & 13.362 & 15.507 & 17.535 & 20.090 & 26.125 \\
9 & 14.684 & 16.919 & 19.023 & 21.666 & 27.877 \\
10 & 15.987 & 18.307 & 20.483 & 23.209 & 29.588 \\
11 & 17.275 & 19.675 & 21.920 & 24.725 & 31.264 \\
12 & 18.549 & 21.026 & 23.337 & 26.217 & 32.910 \\
13 & 19.812 & 22.362 & 24.736 & 27.688 & 34.528 \\
14 & 21.064 & 23.685 & 26.119 & 29.141 & 36.123 \\
15 & 22.307 & 24.996 & 27.488 & 30.578 & 37.697 \\
16 & 23.542 & 26.296 & 28.845 & 32.000 & 39.252 \\
17 & 24.769 & 27.587 & 30.191 & 33.409 & 40.791 \\
18 & 25.989 & 28.869 & 31.526 & 34.805 & 42.312 \\
19 & 27.204 & 30.144 & 32.852 & 36.191 & 43.820 \\
20 & 28.412 & 31.410 & 34.170 & 37.566 & 45.315 \\
21 & 29.615 & 32.671 & 35.479 & 38.932 & 46.797 \\
22 & 30.813 & 33.924 & 36.781 & 40.289 & 48.268 \\
23 & 32.007 & 35.172 & 38.076 & 41.638 & 49.729 \\
\hline
\end{tabular}

Table 4. Selected critical values of $F$-distribution for $\alpha=0.1$

\begin{tabular}{lllllllll}
\hline & $m=3$ & $m=4$ & $m=5$ & $m=6$ & $m=7$ & $m=8$ & $m=9$ & $m=10$ \\
\hline$n=3$ & 5.391 & 5.343 & 5.309 & 5.285 & 5.266 & 5.253 & 5.240 & 5.230 \\
$n=4$ & 4.191 & 4.107 & 4.051 & 4.010 & 3.979 & 3.955 & 3.936 & 3.920 \\
$n=5$ & 3.620 & 3.520 & 3.453 & 3.404 & 3.368 & 3.339 & 3.316 & 3.297 \\
$n=6$ & 3.289 & 3.181 & 3.107 & 3.055 & 3.014 & 2.983 & 2.958 & 2.937 \\
$n=7$ & 3.074 & 2.961 & 2.883 & 2.827 & 2.785 & 2.752 & 2.725 & 2.702 \\
$n=8$ & 2.924 & 2.806 & 2.726 & 2.668 & 2.624 & 2.589 & 2.561 & 2.538 \\
$n=9$ & 2.813 & 2.693 & 2.611 & 2.551 & 2.505 & 2.469 & 2.440 & 2.416 \\
$n=10$ & 2.728 & 2.605 & 2.522 & 2.461 & 2.414 & 2.377 & 2.347 & 2.323 \\
\hline
\end{tabular}

Table 5. Selected critical values of $F$-distribution for $\alpha=0.05$

\begin{tabular}{lllllllll}
\hline & $m=3$ & $m=4$ & $m=5$ & $m=6$ & $m=7$ & $m=8$ & $m=9$ & $m=10$ \\
\hline$n=3$ & 9.278 & 9.117 & 9.013 & 8.941 & 8.887 & 8.845 & 8.812 & 8.786 \\
$n=4$ & 6.592 & 6.388 & 6.256 & 6.163 & 6.094 & 6.041 & 5.999 & 5.964 \\
$n=5$ & 5.410 & 5.192 & 5.050 & 4.950 & 4.876 & 4.818 & 4.779 & 4.735 \\
$n=6$ & 4.757 & 4.534 & 4.387 & 4.284 & 4.207 & 4.148 & 4.099 & 4.060 \\
$n=7$ & 4.347 & 4.120 & 3.971 & 3.866 & 3.787 & 3.726 & 3.677 & 3.637 \\
$n=8$ & 4.066 & 3.838 & 3.687 & 3.581 & 3.500 & 3.438 & 3.388 & 3.347 \\
$n=9$ & 3.863 & 3.633 & 3.482 & 3.374 & 3.293 & 3.230 & 3.179 & 3.137 \\
$n=10$ & 3.708 & 3.478 & 3.326 & 3.217 & 3.136 & 3.072 & 3.020 & 2.978 \\
\hline
\end{tabular}


Table 6. Selected critical values of $F$-distribution for $\alpha=0.025$

\begin{tabular}{lllllllll}
\hline & $m=3$ & $m=4$ & $m=5$ & $m=6$ & $m=7$ & $m=8$ & $m=9$ & $m=10$ \\
\hline$n=3$ & 15.442 & 15.101 & 14.884 & 14.734 & 14.625 & 14.540 & 14.473 & 14.419 \\
$n=4$ & 9.980 & 9.605 & 9.364 & 9.197 & 9.074 & 8.980 & 8.905 & 8.844 \\
$n=5$ & 7.764 & 7.388 & 7.146 & 6.978 & 6.854 & 6.757 & 6.681 & 6.619 \\
$n=6$ & 6.599 & 6.227 & 5.987 & 5.820 & 5.696 & 5.600 & 5.523 & 5.461 \\
$n=7$ & 5.890 & 5.523 & 5.285 & 5.119 & 4.996 & 4.899 & 4.823 & 4.761 \\
$n=8$ & 5.416 & 5.053 & 4.817 & 4.652 & 4.529 & 4.433 & 4.357 & 4.295 \\
$n=9$ & 5.079 & 4.718 & 4.484 & 4.320 & 4.197 & 4.102 & 4.026 & 3.964 \\
$n=10$ & 4.826 & 4.468 & 4.236 & 4.072 & 3.950 & 3.855 & 3.779 & 3.717 \\
\hline
\end{tabular}

Table 7. Selected critical values of $F$-distribution for $\alpha=0.01$

\begin{tabular}{lllllllll}
\hline & $m=3$ & $m=4$ & $m=5$ & $m=6$ & $m=7$ & $m=8$ & $m=9$ & $m=10$ \\
\hline$n=3$ & 29.468 & 28.710 & 28.235 & 27.910 & 27.682 & 27.490 & 27.346 & 27.229 \\
$n=4$ & 16.700 & 15.977 & 15.521 & 15.123 & 14.980 & 14.799 & 14.659 & 14.546 \\
$n=5$ & 12.062 & 11.392 & 10.966 & 10.672 & 10.456 & 10.290 & 10.158 & 10.051 \\
$n=6$ & 9.783 & 9.148 & 8.745 & 8.466 & 8.260 & 8.102 & 7.976 & 7.874 \\
$n=7$ & 8.453 & 7.847 & 7.460 & 7.191 & 6.993 & 6.840 & 6.719 & 6.620 \\
$n=8$ & 7.592 & 7.006 & 6.632 & 6.371 & 6.178 & 6.029 & 5.911 & 5.814 \\
$n=9$ & 6.993 & 6.422 & 6.056 & 5.802 & 5.615 & 5.467 & 5.351 & 5.257 \\
$n=10$ & 6.553 & 5.994 & 5.636 & 5.386 & 5.200 & 5.057 & 4.942 & 4.849 \\
\hline
\end{tabular}

